by the observation that the most sensitive clinical test for detecting occult metastases is the uptake of $\left[{ }^{18} \mathrm{~F}\right]$ fluorodeoxyglucose as determined by PET (18). Can a characteristic that is so useful for diagnosis also be exploited for therapy? Research that elucidated the mechanisms by which cancer cells induce blood vessel growth led to the development of angiogenesis inhibitors as anticancer agents over the last decade (19). Inhibitors of HIF-1, LDHA, PDK1, CA9, NHE1, and now MCT1 have shown anticancer effects in tumor xenograft models $(11,17,20)$. It is likely that over the next decade, clinical translation of advances in our understanding of cancer metabolism will have an impact on cancer therapy. Furthermore, a treatment strategy in which tumors are deprived of both $\mathrm{O}_{2}$ (through angiogenesis inhibition) and their ability to adapt to hypoxia (through metabolic inhibition) may represent a formidable combination therapy.

\section{Acknowledgments}

Work in the author's laboratory is supported by the American Diabetes Association; NIH grants R01-HL55338, P20-GM78494, P01HL65608, and N01-HV28180; and the Johns Hopkins Institute for Cell Engineering.
Address correspondence to: Gregg L. Semenza, 733 North Broadway, Suite 671, Baltimore, Maryland 21205, USA. Fax: (443) 287-5618; E-mail: gsemenza@jhmi.edu.

1. Vaupel, P., and Mayer, A. 2007. Hypoxia and cancer: significance and impact on clinical outcome. Cancer Metastasis Rev. 26:225-239.

2. Helmlinger, G., Yuan, F., Dellian, M., and Jain, R.K. 1997. Interstitial $\mathrm{pH}$ and $\mathrm{pO} 2$ gradients in solid tumors in vivo: high-resolution measurements reveal a lack of correlation. Nat. Med. 3:177-182.

3. Semenza, G.L., et al. 1996. Hypoxia response elements in the aldolase A, enolase 1 , and lactate dehydrogenase A gene promoters contain essential binding sites for hypoxia-inducible factor 1. J. Biol. Chem. 271:32529-32537.

4. Kim, J.W., Tchernyshyov, I., Semenza, G.L., and Dang, C.V. 2006. HIF-1-mediated expression of pyruvate dehydrogenase kinase: a metabolic switch required for cellular adaptation to hypoxia. Cell Metab. 3:177-185.

5. Papandreou, I., Cairns, R.A., Fontana, L., Lim, A.L., and Denko, N.C. 2006. HIF-1 mediates adaptation to hypoxia by actively downregulating mitochondrial oxygen consumption. Cell Metab. 3:187-197.

6. Zhang, H., et al. 2008. Mitochondrial autophagy is a HIF-1-dependent adaptive metabolic response to hypoxia. J. Biol. Chem. 283:10892-10903.

7. Ebert, B.L., Firth, J.D., and Ratcliffe, P.J. 1995. Hypoxia and mitochondrial inhibitors regulate expression of glucose transporter-1 via distinct cisacting sequences. J. Biol. Chem. 270:29083-29089.

8. Wykoff, C.C., et al. 2000. Hypoxia-inducible expression of tumor-associated carbonic anhydrases. Cancer Res. 60:7075-7083.

9. Ullah, M.S., Davies, A.J., and Halestrap, A.P. 2006 The plasma membrane lactate transporter MCT4, but not MCT1, is up-regulated by hypoxia through a HIF-1alpha-dependent mechanism. J. Biol. Chem. 281:9030-9037.

10. Shimoda, L.A., Fallon, M., Pisarcik, S., Wang, J., and Semenza, G.L. 2006. HIF-1 regulates hypoxic induction of NHE1 expression and alkalinization of intracellular $\mathrm{pH}$ in pulmonary arterial myocytes. Am. J. Physiol. Lung Cell Mol. Physiol. 291:L941-L949.

11. Sonveaux, P., et al. 2008. Targeting lactate-fueled respiration selectively kills hypoxic tumor cells in mice. J. Clin. Invest. 118:3930-3942.

12. Seagroves, T.N., et al. 2001. Transcription factor HIF-1 is a necessary mediator of the Pasteur effect in mammalian cells. Mol. Cell. Biol. 21:3436-3444.

13. Yun, Z., Maecker, H.L., Johnson, R.S., and Giaccia, A.J. 2002. Inhibition of PPAR gamma 2 gene expression by the HIF-1-regulated gene DEC1/Stra13: a mechanism for regulation of adipogenesis by hypoxia. Dev. Cell. 2:331-341.

14. Liao, D., and Johnson, R.S. 2007. Hypoxia: a key regulator of angiogenesis in cancer. Cancer Metastasis Rev. 26:281-290.

15. Cárdenas-Navia, L.I., et al. 2008. The pervasive presence of fluctuating oxygenation in tumors. Cancer Res. 68:5812-5819.

16. Deberardinis, R.J., Sayed, N., Ditsworth, D., and Thompson, C.B. 2008. Brick by brick: metabolism and tumor cell growth. Curr. Opin. Genet. Dev. 18:54-61.

17. Kroemer, G., and Pouyssegur, J. 2008. Tumor cell metabolism: cancer's Achilles' heel. Cancer Cell. 13:472-482.

18. Gatenby, R.A., and Gillies, R.J. 2004. Why do cancers have high aerobic glycolysis? Nat. Rev. Cancer. 4:891-899.

19. Kerbel, R.S. 2008. Tumor angiogenesis. N. Engl. J. Med. 358:2039-2049.

20. Melillo, G. 2007. Targeting hypoxia cell signaling for cancer therapy. Cancer Metastasis Rev. 26:341-352.

\title{
Autophagy-induced tumor dormancy in ovarian cancer
}

\author{
Ravi K. Amaravadi \\ Department of Medicine and Abramson Cancer Center, University of Pennsylvania, Philadelphia, Pennsylvania, USA.
}

\begin{abstract}
Autophagy - a process of "self-eating" that involves enzymatic digestion and recycling of cellular constituents in response to stress - contributes to both cancer cell death and survival. In this issue of the JCI, Lu et al. report that controlled induction of tumor suppressor gene aplasia Ras homolog member I $(A R H I)$ results in autophagic cell death of human ovarian cancer cells in vitro (see the related article, doi:10.1172/JCI35512). However, within xenograft tumors in mice, multiple factors within the tumor microenvironment switched ARHI-induced autophagy to a mechanism of tumor cell survival, leading to tumor dormancy. Since ARHI expression is suppressed in the majority of breast and ovarian cancers but is high in premalignant lesions, ARHI-induced autophagy could be manipulated for therapeutic benefit.
\end{abstract}

Nonstandard abbreviations used: ARHI, aplasia Ras homolog member I; BECN1, beclin 1.

Conflict of interest: The author has declared that no conflict of interest exists.

Citation for this article: J. Clin. Invest. doi:10.1172/ JCI37667.
Cells that encounter a variety of stresses undergo an evolutionarily conserved process of self-digestion termed autophagy. The importance of this intracellular damage response for pathophysiology has been established across multiple fields, includ- ing infectious disease, neurodegeneration, heart failure, and cancer (1). In cancer in particular, the debate continues to rage as to whether or not autophagy is primarily a mechanism of cell death or cell survival. This is important to understand in order to preferentially promote the clinical development of therapeutic interventions that can either inhibit or enhance autophagy in tumor cells. A number of clinically available cancer therapeutics, including DNAdamaging chemotherapy, radiation therapy, and molecularly targeted therapies have been found to induce autophagy in cell culture and animal models (2). Recent investigations have found that inhibition of therapy-induced autophagy with chloroquine derivatives can enhance cell death in estab- 
A In vitro

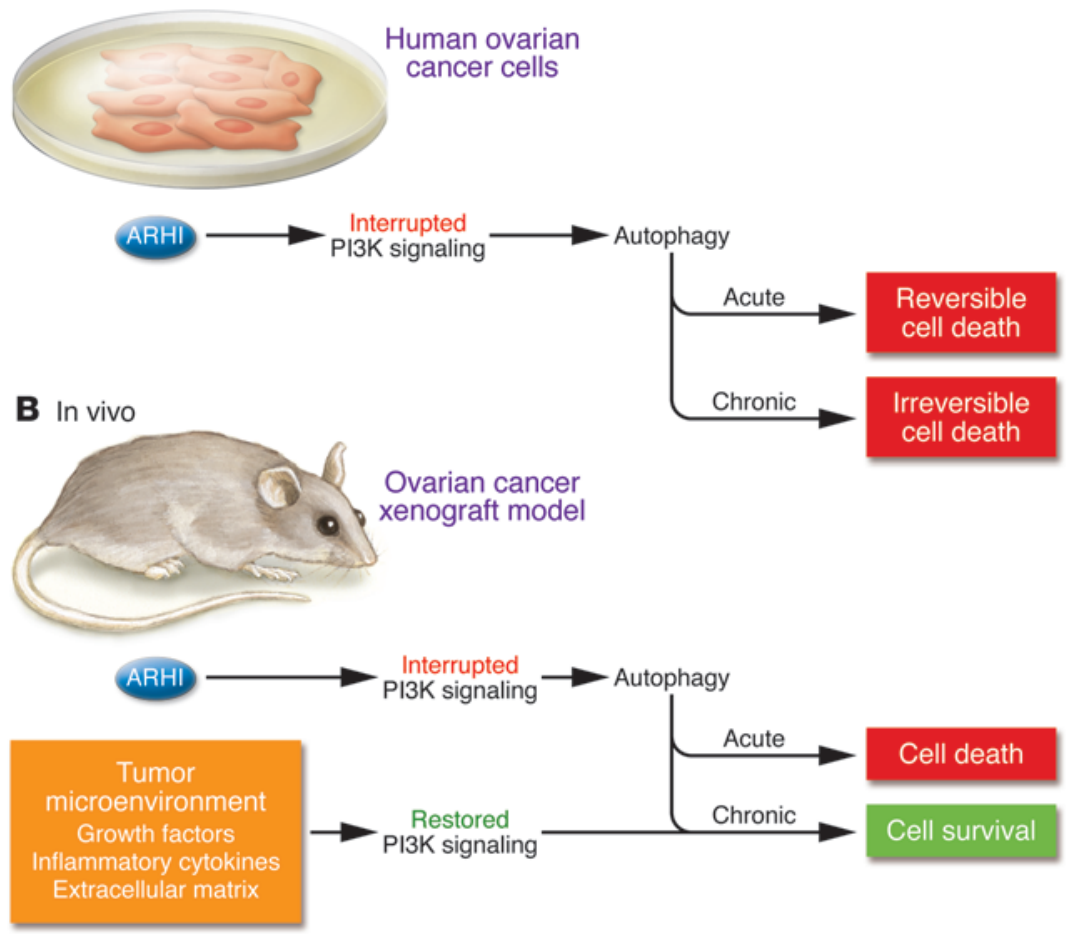

\section{Figure 1}

Consequences of forced expression of the tumor suppressor gene $A R H I$ in ovarian cancer. In their study in this issue of the $\mathrm{JCl}$, Lu et al. (8) report that controlled expression of the tumor suppressor gene $A R H I$ in human ovarian cancer cells leads to autophagy. (A) ARHI expression was associated with interrupted PI3K signaling, which resulted in reversible (under conditions of transient ARHI expression) or irreversible (under conditions of chronic $\mathrm{ARHI}$ expression) autophagic cell death in vitro. (B) In contrast, ARHI-induced autophagy contributed to cell survival and tumor dormancy in ovarian cancer xenografts in mice, due to partially restored $\mathrm{PI} 3 \mathrm{~K}$ signaling from elements of the tumor microenvironment. lished tumors, leading to enhanced tumor regression and delayed tumor regrowth (3). In this study, a combination of experiments involving RNA interference and drugs were used to demonstrate that therapy-induced autophagy in established tumors allows tumor cells to survive therapeutic stress (3). The cytoprotective role of autophagy following chemotherapy has been confirmed by other investigators (4-6). These findings have led to a growing number of early-phase clinical trials that aim to establish the safety and preliminary efficacy of combining agents that induce autophagy with hydroxychloroquine in patients with advanced malignancies refractory to standard treatments. Collectively, these actively accruing clinical trials represent the first attempt to manipulate autophagy for therapeutic benefit in cancer.

Autophagy inhibition with chloroquine has also been found to significantly delay the onset of spontaneous tumors in a transgenic mouse model of Myc-induced lymphoma (7). This study suggested that autophagy inhibition may have a role not only in combination therapies for patients with active metastatic disease, but also in the prevention of early disease. However, conducting a clinical trial of a novel autophagy inhibitor, or in the case of hydroxychloroquine, an old drug being used for new indication, in tumor-free patients is difficult, requiring large numbers of patients, and long periods of followup. While the known safety and tolerability of chloroquine derivatives suggest they may be ideal drugs to test as chemopreventative agents, committing the resources necessary to conduct prevention trials may not be warranted until a potential benefit of this strategy has been established in a clinical setting with short-term outcomes, such as in metastatic disease. Another clinical setting that could be more feasible to investigate autophagy inhibitors is in the setting of minimal residual disease with high risk of relapse. A common problem across multiple malignancies is that cancer therapy can initially result in a complete remission, but there is an extremely high chance of relapse. This indicates there are clinically undetectable tumor cells that are dormant and have the ability to regrow at a later date. This is an ideal setting to investigate the role of autophagy and the effects of pharmacological autophagy inhibitors.

In this issue of the JCI, Lu, Bast, and colleagues report an ovarian cancer model of minimal residual disease and identify a role for autophagy in supporting tumor dormancy (8). Additionally, their analysis of the effects of controlled expression of the autophagy-inducing tumor suppres- sor gene aplasia Ras homolog member I $(A R H I)$ in human ovarian cancer cells in vitro versus in vivo sheds light on the relationship between autophagic cell death and autophagic cell survival and implicates an underappreciated role for the tumor microenvironment in deciding this fate.

\section{The ras homolog ARHI activates autophagy in ovarian cancer}

Over the past decade, Bast and colleagues have extensively characterized the genetic and biochemical underpinnings of ARHI, a maternally imprinted Ras-homologous kinase that exerts growth-inhibitory effects when expressed in ovarian cancer cells (9). ARHI is expressed in normal ovarian epithelium, but through the transition to malignancy, ARHI expression is suppressed in a stepwise fashion. Examination of tumor samples from cancer patients has determined that ARHI loss of heterozygosity ( $\mathrm{LOH}$ ) occurs in $60 \%-70 \%$ of ovarian and breast cancers (10-12). Another gene whose expression is suppressed through the $\mathrm{LOH}$ in breast and ovarian cancer is the autophagy-associated gene beclin 1 (BECN1). Unlike BECN1, in which the wildtype allele is retained and therefore can be expressed (13), the retained allele of ARHI is maternally imprinted and therefore not expressed (14). Since there appears to be 


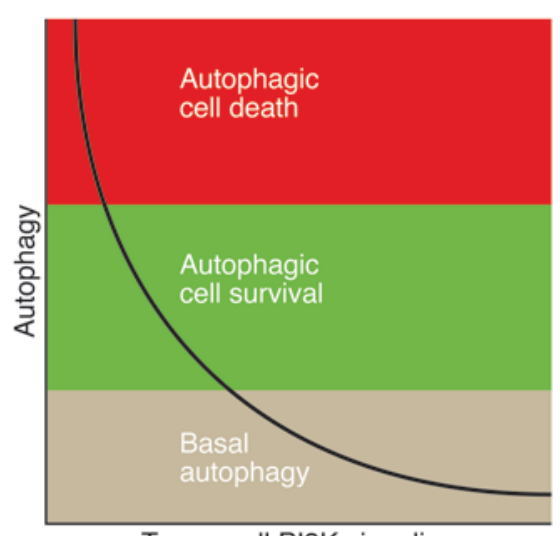

Tumor cell PI3K signaling

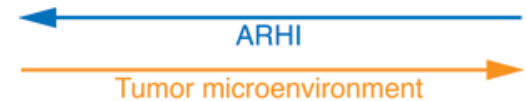

Figure 2

The magnitude of autophagy may determine the fate of cancer cells in vivo. This conceptual model proposes a relationship between the magnitude of cancer cell autophagy and its consequences on cell death and cell survival. As reported by Lu et al., expression of the tumor suppressor gene $A R H I$ suppresses signal transduction through $\mathrm{PI} 3 \mathrm{~K}$ signaling, while factors within the tumor microenvironment maintain $\mathrm{PI} 3 \mathrm{~K}$ signaling within tumor cells (8). The magnitude of autophagy may be inversely proportional to PI3K signaling, and thresholds may exist within cancer cells for the magnitude of autophagy that leads to cell death versus the magnitude of autophagy that leads to cell survival.

a selective advantage to complete loss of ARHI expression during neoplastic transformation, developing a tumor model that allows inducible expression of ARHI at physiological levels in established tumors has provided valuable insight into the ARHI-mediated mechanism of tumor suppression. The insight gained from this current study by Lu et al. (8) has the potential to launch clinical investigations aimed at changing therapeutic approaches to the treatment of ovarian and breast cancers.

Using doxycycline-inducible expression of ARHI, the authors demonstrated that stable or transient physiologic expression of ARHI in human ovarian cancer cell lines and other human cancer cell lines resulted in the induction of autophagy and not apoptosis (8). Elegant in-cell cross-linking studies demonstrated that ARHI localizes with the autophagy marker light chain 3 (LC3) on the surface of autophagic vesicles, providing evidence that ARHI may regulate autophagy though direct physical interaction with the autophagic machinery. Expression of ARHI was associated with increased expression of ATG4, which is encoded by one of the core autophagy genes (ATG4) in a group of genes denoted as ATGs. This oxidant-sensitive enzyme deconjugates LC3 from lipids on the surface of autophagic vesicles. To look for more indirect means by which ARHI could regulate autophagy, the authors used reverse-phase protein microarray analysis to look at multiple signaling events simultaneously. Expression of ARHI lead to a marked inhibition of PI3K/AKT/mammalian target of rapamycin (PI3K/AKT/ mTOR) and MAPK signaling. Expression of the mutant ARHI that was missing the $\mathrm{N}$-terminal domain, which distinguishes its structure from other Ras family proteins, prevented growth factor-induced membrane recruitment of AKT and limited the activation of downstream effectors mTOR and $\mathrm{p} 70 \mathrm{~S} 6 \mathrm{~K}$.

\section{Death or survival \\ from ARHI-induced autophagy}

To investigate the functional consequences of ARHI-induced autophagy, the authors determined that ARHI expression prevented clonogenic growth after one week and resulted in cell death after two weeks (8). In contrast to a previous study in which chronic growth factor withdrawal from apoptosis-deficient cells resulted in a reversible form of autophagy that contributed to cell survival (15), Lu et al. show that transient expression of ARHI in human ovarian cancer cells leads to a reversible form of autophagy, but that chronic ARHI expression leads to irreversible autophagic cell death (Figure 1A). When ARHI expression was induced in ovarian cancer xenografts in mice, these authors found that acute cell death ensued, leading to tumor regression. However, removal of doxycycline, which resulted in the absence of ARHI expression, led to tumor regrowth. These dormant cells were likely dependant on autophagy as a survival strategy, since systemic pretreatment of mice harboring ARHI-suppressed ovarian xenografts with the autophagy inhibitor chloroquine resulted in a significant delay of tumor regrowth once ARHI expression was downregulated.

These results suggest that, unlike the in vitro experiments, in which cells exposed to ARHI were destined for autophagic cell death, within the tumor microenvironment, ARHI-induced autophagy allows a cohort of hibernating cells to survive and eventually recur as tumors once the ARHI tumor suppressor is downregulated (8). Lu et al. investigated the contributions of growth factors, inflammatory cytokines, angiogenic factors, and extracellular matrix proteins to autophagic cell death in vitro and in vivo. They found that, by limiting the ARHI-dependent suppression of intracellular PI3K signaling, members of each of these classes of molecules within the tumor microenvironment can contribute to changing autophagy from a cell death mechanism to a cell survival mechanism. Taken together, these results suggest that the magnitude of autophagy is inversely related to PI3K signaling. ARHI suppresses intracellular PI3K signaling, and elements of the tumor microenvironment promote intracellular PI3K signaling. Autophagic cell death occurs when autophagy is maximally induced, but when levels of autophagy are elevated above basal levels, but are less than maximum levels, the result is autophagic cell survival (Figure 2).

\section{Tumor suppressor genes and autophagy}

This microenvironment-directed switch may also shed light on why a gene whose expression can suppress multiple signaling pathways and lead to cell death is expressed constitutively in normal and premalignant epithelium. The role of autophagy in limiting DNA damage and genomic instability has been established (16). One hypothesis that can be tested in future studies is that ARHI-induced autophagy limits the rate of genomic instability and neoplastic transformation, while microenvironmental signals in normal or premalignant native tissue simultaneously limit ARHI-induced autophagic cell death. A related question that arises is whether the effects of ARHI expression are specific to ovarian and breast cancer, or whether ARHI expression is selectively suppressed in other cancers. Similarly, what is the interaction between ARHI expression and common mutations in oncogenes encountered in ovarian cancer? The SKOv3 ovarian cancer cell line has constitutively activated PI3K/AKT signaling but no known mutations in $P I 3 K$, phosphatase and tensin homolog (PTEN), RAS, or BRAF. Would the effects of ARHI expression be different in tumor cells harboring mutations in PI3K, RAS, or BRAF? Finally, ARHI can be added to a growing list of "non- $A T G$ " genes that were not originally identified as autophagy genes but that directly regulate this pro- 
cess through physical engagement of the autophagic machinery. This list includes, but is not limited to, endophilin B1 (BIF1) (17), UV radiation resistance-associated gene (UVRAG) (17), p62 (18), and WD repeat domain phosphoinositide-interacting 1 (WIPI1) (19). Individually, aberrant expression of some of these genes has been demonstrated in a wide variety of malignancies. It remains to be determined whether more or all of these genes are bonafide tumor suppressor genes in human tumors. It will also be important to determine whether the genetic aberrations in multiple autophagy-associated genes can be found in the same tumor, as well as the implications of this for tumorigenesis and clinical prognosis.

\section{Clinical implications of the inducible-ARHI tumor model}

Regardless of these remaining questions, the inducible ARHI tumor model reported here (8) also has practical implications in understanding the role of autophagy in the treatment of ovarian cancer patients. The authors point out that $60 \%$ of primary ovarian tumors have decreased ARHI expression associated with shortened disease-free survival after chemotherapy. This observation could be explained by a propensity for early recurrence in ovarian tumors lacking ARHI-induced autophagy, which suggests that disease-free survival could be prolonged in this subgroup of patients by combining drugs known to induce autophagy with current chemotherapy regimens. Alternatively, in patients whose tumors express ARHI, could the addition to standard chemotherapy of an autophagy inhibitor prolong disease-free survival compared with chemotherapy alone? Examining the tumor tissue of
ARHI-positive and ARHI-negative tumors for markers of autophagy is a first step toward determining whether these clinical investigations should be launched. A parallel preclinical effort would be to exploit this tumor model as a platform to validate autophagy inhibitors with pharmacological properties identified in drug screens. Promising autophagy inhibitors should reproducibly delay or prevent regrowth of ARHI-supressed tumors upon removal of doxycycline. While most tumor suppressor genes are not druggable targets themselves, the current study by Lu et al. (8) demonstrates that mechanistic studies of tumor suppressor genes can uncover druggable targets that can be tested in defined subsets of cancer patients.

\section{Acknowledgments}

The author is supported by NIH grant 1K23CA120862.

Address correspondence to: Ravi K. Amaravadi, Abramson Cancer Center, University of Pennsylvania, 12 Penn Tower, 3400 Spruce Street, Philadelphia, Pennsylvania 19104, USA. Phone: (215) 662-7402; Fax: (215) 662-7865; E-mail: ravi.amaravadi@ uphs.upenn.edu.

1. Mizushima, N., Levine, B., Cuervo, A.M., and Klionsky, D.J. 2008. Autophagy fights disease through cellular self-digestion. Nature. 451:1069-1075.

2. Amaravadi, R.K., and Thompson, C.B. 2007. The roles of therapy-induced autophagy and necrosis in cancer treatment. Clin. Cancer Res. 13:7271-7279.

3. Amaravadi, R.K., et al. 2007. Autophagy inhibition enhances therapy-induced apoptosis in a Myc-induced model of lymphoma. J. Clin. Invest. 117:326-336

4. Carew, J.S., et al. 2007. Targeting autophagy augments the anticancer activity of the histone deacetylase inhibitor SAHA to overcome Bcr-Abl-mediated drug resistance. Blood. 110:313-322.

5. Katayama, M., Kawaguchi, T., Berger, M.S., and Pieper, R.O. 2007. DNA damaging agent-induced autophagy produces a cytoprotective adenosine triphosphate surge in malignant glioma cells. Cell Death Differ. 14:548-558.

6. Abedin, M.J., Wang, D., McDonnell, M.A., Lehmann, U., and Kelekar, A. 2007. Autophagy delays apoptotic death in breast cancer cells following DNA damage. Cell Death Differ. 14:500-510.

7. Maclean, K.H., Dorsey, F.C., Cleveland, J.L., and Kastan, M.B. 2008. Targeting lysosomal degradation induces p53-dependent cell death and prevents cancer in mouse models of lymphomagenesis. J. Clin. Invest. 118:79-88.

8. Lu, Z., et al. 2008. The tumor suppressor gene ARHI regulates autophagy and tumor dormancy in human ovarian cancer cells. J. Clin. Invest. 118:3917-3929.

9. Yu, Y., et al. 2006. Biochemistry and biology of ARHI (DIRAS3), an imprinted tumor suppressor gene whose expression is lost in ovarian and breast cancers. Methods Enzymol. 407:455-468.

10. Yu, Y., et al. 1999. NOEY2 (ARHI), an imprinted putative tumor suppressor gene in ovarian and breast carcinomas. Proc. Natl. Acad. Sci. U. S. A. 96:214-219.

11. Rosen, D.G., et al. 2004. Expression of the tumor suppressor gene ARHI in epithelial ovarian cancer is associated with increased expression of p21WAF1/CIP1 and prolonged progression-free survival. Clin. Cancer Res. 10:6559-6566.

12. Wang, L., et al. 2003. Loss of the expression of the tumor suppressor gene ARHI is associated with progression of breast cancer. Clin. Cancer Res. 9:3660-3666.

13. Aita, V.M., et al. 1999. Cloning and genomic organization of beclin 1, a candidate tumor suppressor gene on chromosome 17q21. Genomics. 59:59-65.

14. Yuan, J., et al. 2003. Aberrant methylation and silencing of ARHI, an imprinted tumor suppressor gene in which the function is lost in breast cancers. Cancer Res. 63:4174-4180.

15. Lum, J.J., et al. 2005. Growth factor regulation of autophagy and cell survival in the absence of apoptosis. Cell. 120:237-248.

16. Mathew, R., et al. 2007. Autophagy suppresses tumor progression by limiting chromosomal instability. Genes Dev. 21:1367-1381.

17. Takahashi, Y., et al. 2007. Bif-1 interacts with Beclin 1 through UVRAG and regulates autophagy and tumorigenesis. Nat. Cell Biol. 9:1142-1151.

18. Pankiv, S., et al. 2007. p62/SQSTM1 binds directly to Atg8/LC3 to facilitate degradation of ubiquitinated protein aggregates by autophagy. J. Biol. Chem. 282:24131-24145.

19. Proikas-Cezanne, T., et al. 2004. WIPI-1alpha (WIPI49), a member of the novel 7-bladed WIPI protein family, is aberrantly expressed in human cancer and is linked to starvation-induced autophagy. Oncogene. 23:9314-9325. 